\title{
LETTER
}

\section{IL-10: a marker of cardiac bypass?}

\author{
Karen Balonze', Giovanna Olivera', Christopher Mendoza², Ashish Tikotekar*3 and Amay Parikh³ \\ See related research by Matera et al., http://ccforum.com/content/17/2/R64
}

We appreciate the efforts of Matera and colleagues' study establishing the utility of IL-10 as a diagnostic and prognostic marker of early sepsis [1]; however, we have some concerns.

Limiting the study to the surgical population limits the generalizability. More importantly, inclusion of 28 (of the 52) patients after on-pump cardiac surgery is concerning. Pump surgery is widely accepted to result in an intense surge in inflammatory markers, including IL-10 [2]. Also, the duration of pump surgery can be variable and the inflammatory response varies with the time spent on pump. The association of IL-10 with a worse prognosis (nonsurvivor group) may therefore not be valid. Cardiac ICU protocols such as the use of perioperative antibiotics were not discussed, which may affect mortality.
In Table 3 of their article, the confidence interval for the odds ratio of IL-10 for the prognosis of bacteremic systemic inflammatory response syndrome patients includes the value 1 [1]. Including this value limits the applicability. The values of biomarkers were not checked daily, and therefore we cannot rule out a new increase in the levels of IL-10 secondary to subsequent episodes of inflammation [3]. The Sequential Organ Failure Assessment score and IL-10 values on day 1 and day 7 correlate with mortality. The utility of an expensive and timeconsuming biomarker is questioned when a simple, quick and bedside test could predict the outcome.

Despite the positive results of the marker, it is difficult to imagine how such information would change management in the era of the surviving sepsis guidelines [4].

\section{Authors' response}

G Matera, R Puccio, A Giancotti, A Quirino, MC Pulicari, E Zicca, S Caroleo, A Renzulli, MC Liberto, A Foca

We thank the editor for the opportunity to reply to the letter by Balonze and colleagues, who reported some concerns regarding our recently published paper [1].

We do not feel our article is limited to the surgical population: indeed, $36.5 \%$ of the enrolled subjects were medical/nonsurgical patients (Table 1 in [1]). We agree that pump surgery results in a substantial increase in inflammatory markers including IL-10 [3]; however, many nonsurgical patients might be subjected to a qualitatively different but comparable stressful and acute event (for example, adult respiratory distress syndrome, shock). The number of nonsurvivors subjected to onpump cardiac surgery was almost the same in comparison with nonsurvivors never subjected to on-pump cardiac surgery. Also, the time spent during the on-pump phase

*Correspondence: tikoteaa@umdnj.edu

${ }^{3}$ Pulmonary and Critical Care Division, Department of Medicine, Robert Wood

Johnson Medical School, 1 Robert Wood Johnson Place, PO Box 19, New

Brunswick, NJ 08903-0019, USA

Full list of author information is available at the end of the article of cardiac surgery was fairly homogeneous for all patients who underwent such a procedure. The antibiotic administration for the surgical patients followed a default protocol based on wide-spectrum antimicrobial agents.

Moreover, for cardiac surgery patients the sampling at day 1 was in accordance with the main source of inflammation (cardiopulmonary bypass/surgery stress) and with the standard clinical course of these patients. However, the noncardiac surgery patients could effectively show a clinical course with multiple episodes of inflammation/infection; regarding these patients, we agree with the comment of Balonze and colleagues.

Clinical features such as the Sequential Organ Failure Assessment score are simply clinical tools that, in our opinion, should be strengthened with the use of biomarkers [3], reflecting both inflammatory and infectious processes.

\author{
Abbreviations \\ $\mathrm{IL}$, interleukin. \\ Competing interests \\ The authors declare that they have no competing interests.
}




\section{Author details}

IInternal Medicine Division, Department of Medicine, Department of Medicine, Robert Wood Johnson Medical School, 1 Robert Wood Johnson Place, PO Box 19, New Brunswick, NJ 08903-0019, USA. ²Department of Emergency Medicine, Robert Wood Johnson Medical School, 1 Robert Wood Johnson Place, New Brunswick, NJ 08903-0019, USA. ${ }^{3}$ Pulmonary and Critical Care Division, Department of Medicine, Robert Wood Johnson Medical School, 1 Robert Wood Johnson Place, PO Box 19, New Brunswick, NJ 08903-0019, USA.

\section{Published: 1 August 2013}

\section{References}

1. Matera G, Puccio R, Giancotti A, Quirino A, Pulicari MC, Zicca E, Caroleo S, Renzulli A, Liberto M, Foca A: Impact of interleukin-10, soluble CD25 and interferon-gamma on the prognosis and early diagnosis of bacteremic systemic inflammatory response syndrome: a prospective observational study. Crit Care 2013, 17:R64.
2. Diegeler A, Doll N, Rauch T, Haberer D, Walther T, Falk V, Gummert J, Autschbach R, Mohr FW: Humoral immune response during coronary artery bypass grafting: a comparison of limited approach, 'off-pump' technique, and conventional cardiopulmonary bypass. Circulation 2000, 102(19 Suppl 3):|||95-|||100.

3. Faix JD: Biomarkers of sepsis. Crit Rev Clin Lab Sci 2013, 50:23-36.

4. Dellinger RP, Levy MM, Rhodes A, Annane D, Gerlach H, Opal SM, Sevransky JE, Sprung CL, Douglas IS, Jaeschke R, Osborn TM, Nunnally ME, Townsend SR, Reinhart K, Kleinpell RM, Angus DC, Deutschman CS, Machado FR, Rubenfeld GD, Webb S, Beale RJ, Vincent JL, Moreno R; Surviving Sepsis Campaign Guidelines Committee including The Pediatric Subgroup: Surviving Sepsis Campaign: international guidelines for management of severe sepsis and septic shock, 2012. Intensive Care Med 2013, 39:165-228.

doi:10.1186/cc12776

Cite this article as: Balonze K, et al.: IL-10: a marker of cardiac bypass? Critical Care 2013, 17:443. 